\title{
High-energy 3D calorimeter for use in gamma-ray astronomy based on position-sensitive virtual Frisch-grid CdZnTe detectors
}

\author{
Alexander Moiseev $^{\mathrm{a} *}$, Alexey Bolotnikov ${ }^{\mathrm{b}}$, GianLuigi DeGeronimo ${ }^{\mathrm{b}}$, Elizabeth \\ Hays $^{c}$, Ralph James ${ }^{\mathrm{d}}$, David Thompson ${ }^{\mathrm{c}}$, and Emerson Vernon ${ }^{\mathrm{b}}$ \\ ${ }^{a}$ CRESST/NASA/GSFC and University of Maryland, College Park, Greenbelt, MD 20771, USA \\ E-mail: alexander.a.moiseevenasa.gov \\ ${ }^{b}$ Brookhaven National Laboratory, Upton, NY 11973, USA \\ ${ }^{c}$ NASA/GSFC, Greenbelt, MD 20771, USA \\ ${ }^{d}$ Savannah River National Laboratory, Savannah River, SC, USA
}

ABSTRACT: We will present a concept for a calorimeter based on a novel approach of 3D positionsensitive virtual Frisch-grid CdZnTe (hereafter CZT) detectors. This calorimeter aims to measure photons with energies from $\sim 100 \mathrm{keV}$ to $20-50 \mathrm{MeV}$. The expected energy resolution at $662 \mathrm{keV}$ is better than $1 \% \mathrm{FWHM}$, and the photon interaction position-measurement accuracy is better than $1 \mathrm{~mm}$ in all 3 dimensions.

Each CZT bar is a rectangular prism with typical cross-section from $5 \times 5$ to $7 \times 7 \mathrm{~mm}^{2}$ and length of 2-4 cm. The bars are arranged in modules of $4 \times 4$ bars, and the modules themselves can be assembled into a larger array. The 3D virtual voxel approach solves a long-standing problem with CZT detectors associated with material imperfections that limit the performance and usefulness of relatively thick detectors (i.e., $>1 \mathrm{~cm}$ ). Also, it allows us to use the standard (unselected) grade crystals, while achieving the energy resolution of the premium detectors and thus substantially reducing the cost of the instrument.

Such a calorimeter can be successfully used in space telescopes that use Compton scattering of $\gamma$ rays, such as AMEGO, serving as part of its calorimeter and providing the position and energy measurement for Compton-scattered photons (like a focal plane detector in a Compton camera). Also, it could provide suitable energy resolution to allow for spectroscopic measurements of $\gamma$ ray lines from nuclear decays.

KEYWORDS: $\gamma$-ray astronomy, CdZnTe detector, position-sensitive calorimeter 


\section{Contents}

1. Introduction $\quad 1$

2. CZT Detectors $\quad 2$

$\begin{array}{ll}2.1 \text { Virtual Frisch-grid detectors } & 3\end{array}$

3. Concept of the Calorimeter $\quad 6$

$\begin{array}{ll}\text { 4. Summary } & 7\end{array}$

$\begin{array}{ll}\text { 5. References } & 7\end{array}$

\section{Introduction}

The $\gamma$-ray energy range from a few hundred $\mathrm{keV}$ to a few hundred $\mathrm{MeV}$ has remained largely unexplored since the pioneering but limited observations by COMPTEL on CGRO (1991-2000). Fundamental astrophysics questions can be addressed by a mission in the $\mathrm{MeV}$ range, from astrophysical jets and extreme physics of compact objects to a large population of unidentified objects.

To address these questions, we are developing AMEGO: All-sky Medium Energy Gamma-ray Observatory, a NASA Probe-class mission, to investigate the energy range from $300 \mathrm{keV}$ to $>10$ $\mathrm{GeV}$ with good energy and angular resolution [1]. For the energy below $\sim 10 \mathrm{MeV}$ its sensitivity approaches a factor of 20-50 better than previous instruments. Measurements at these energies are challenging, mainly due to the specifics of photon detection: Compton scattering and pair production compete. These two interaction processes require different approaches in both detection and data analysis, and consequently in the instrument concept.

Event types for a Compton-Pair telescope are shown in Fig.1. Detection of a pair event requires accurate tracking of the electron and positron and measurement of their energy. The detection of Compton-scattered photons is more complicated and, in addition to the measurement of the point of the first interaction, requires accurate measurement of the direction and energy of the scattered photon. This requires the "Calorimeter" schematically shown in Fig.1 to have good energy and position resolution. A calorimeter based on position-sensitive CZT detectors can be a very promising detector for such an instrument.

We are developing a three-dimensional, high-energy-resolution, high-efficiency calorimeter for photon energies $0.2-10 \mathrm{MeV}$, based on novel drift (Frisch-grid) bar CdZnTe (hereafter CZT) detectors recently developed at Brookhaven National Laboratory $[2,3]$. This calorimeter will have a position resolution of $<1 \mathrm{~mm}$ along each of 3 axes and energy resolution of $1-3 \%$, depending on the incident photon energy, with capability of operating in ambient conditions. The design features will allow us to correct the response non-uniformity caused by crystal defects, which means that standard grade (unselected) CZT material can be used. This will significantly increase 
the yield of acceptable detectors and consequently reduce the cost of the instrument. This technology represents a breakthrough, because it promises high performance with large-area detectors at a reasonable cost.

\section{CZT Detectors}

CZT is a promising medium for roomtemperature $\gamma$-ray detectors and has several advantages over other semiconductor detectors (for example, $\mathrm{Si}$ or $\mathrm{Ge}$ ): a high atomic number, high density (important for $\gamma$-ray detection), a low Fano-factor of 0.1 , ability to operate at room temperature, and chemical and mechanical stability. The operational principle of CZT detectors is very similar to classic gas ionization chambers and to other semiconductor detectors. CZT detectors have been used in a number of space experiments, e.g. Swift, InFocus, and NuStar. The best energy resolution, approaching the electro-hole

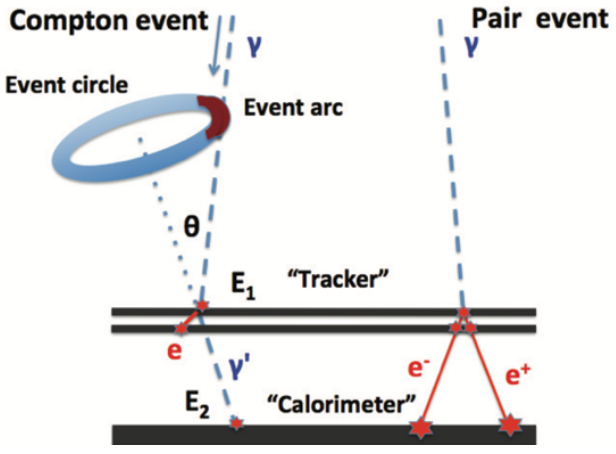

Figure 1. Event types for Compton-Pair telescopes production statistic limit, $0.5 \%$ FWHM at 662

$\mathrm{keV}$, has been demonstrated with large-volume pixelated detectors, up to $6 \mathrm{~cm}^{3}$, made from the highest-quality CZT crystals [4]. The main challenge facing detector developers today is not how to design high-performance CZT detectors but how to make them less expensive and more widely available for practical applications. The main obstacle is the response non-uniformities caused by crystal defects that are present even in the best-quality commercial CZT material [5].

The low production yield of high quality crystals hampers the use of CZT detectors for $\gamma$-ray spectroscopy. Significant efforts have been directed towards improving the quality of CZT crystals to make them generally available for radiation detectors. Today, the most common defects still remaining in detector-grade CZT crystals are subgrain boundaries and Te inclusions or dislocation clusters left after annealing Te inclusions, which result in local variations in distributions of carriers trapping centers. These defects affect both the energy resolution and photopeak efficiency. The production yield of acceptable crystals is governing today the cost and availability of CZT detectors. It is clear that probability of finding "good" crystals decreases with volume, and it also depends on detector geometry.

One way to address this problem is to implement detector designs that would allow vendors to increase production yields and lower detector costs. Another possibility is to apply accurate and predictable correction of the response for the non-uniformities caused by the small defects. The latter approach is justified by the fact that the defect locations inside crystals are fixed, while the signal variations come about due to random distributions of interaction points and, thus, can be corrected by dividing the detector's active volume into sufficiently small voxels and equalizing responses from all voxels. Such detectors, called high-granularity position-sensitive detectors, can significantly improve the performance of CZT detectors fabricated from CZT crystals with wider acceptance boundaries, leading to an increase of their availability and expected decrease in cost. 
Several types of CZT detectors were shown to achieve energy resolution close to the CZT material intrinsic limit determined by the statistics of the electron-hole pair production. Two detector designs: pixelated and coplanar-grid are particularly suitable for large-volume detectors. However, their performances were also shown to strongly depend on the quality of CZT crystals. Indeed, while the co-planar grid detector (CPG) device can offer drift-time (or interaction depth) correction, it has no position-sensitivity in XY directions, which means that the variations in the charge-collection efficiency over the device's area may degrade the spectral response. 3D devices have an advantage over CPG because they can correct large-scale response variations in 3-D coordinate space. However, because of rather coarse segmentations, they still rely on using the highest-quality CZT crystals.

\subsection{Virtual Frisch-grid detectors}

The proposed calorimeter is based on arrays of position-sensitive virtual Frischgrid (PSVFG) detectors recently introduced

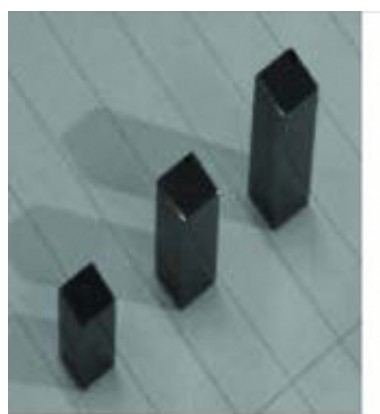

Figure 2. $6 \times 6 \mathrm{~mm}^{2} \mathrm{CZT}$ bars of different length (left) and schematic of the detector (right).

by Brookhaven National Laboratory for imaging and spectroscopy of $\gamma$-ray sources. The array employs small area, currently $6 \times 6 \mathrm{~mm}^{2}$ (can be larger), but long, potentially up to 3-4 $\mathrm{cm}$ CZT crystals (bars) tightly packaged together. Each crystal is furnished with two gold contacts on the top and bottom surfaces (the anode and the cathode) and encapsulated inside an ultra-thin dielectric shell (or coating), which electrically insulates and mechanically protects the crystals. To make the detector position-sensitive, 4 metal pads are attached to the shell near the anodes (Fig. 2). The combination of high geometrical aspect ratio of the detector and the virtually grounded pads produces a strong shielding effect as if a real Frisch-grid were placed inside the crystal to shield the anode. Although, the virtual Frisch-grid design was originally proposed for a noble gas filled drift cells [6], this idea turned out to be more successful when applied to CZT detectors: CAPture ${ }^{\mathrm{TM}}$ [7], hemispherical [8], Frisch-ring [9], and capacitive Frisch-grid [10].

The amplitudes of the signals read out from the pads are used to evaluate $\mathrm{X}-\mathrm{Y}$ coordinates, while the drift time and the cathode-to-anode ratio, $\mathrm{C} / \mathrm{A}$, are used to independently evaluate the $\mathrm{Z}$ coordinate for the location of each interaction point. However, the design constraints require us to connect together the pads of adjacent detectors facing each other. Furthermore, we connect together the cathodes of 4 adjacent detectors. This also reduces the total number of readout channels, but it creates signal ambiguity when multiple interactions occur in adjacent detectors. In such cases, we cannot use the pads with signals generated by electron clouds in two adjacent but different detectors. The remaining signals from two orthogonal or three pads still can be used to evaluate the positions of the interaction points. We verified experimentally that two signals from two

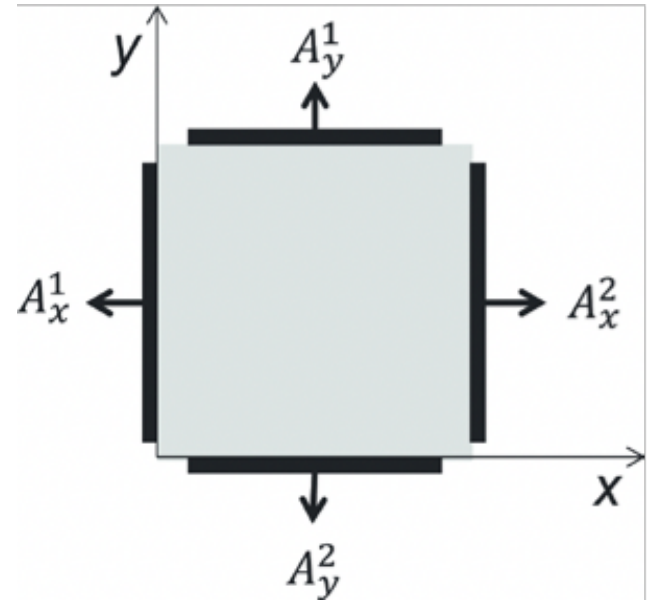

Figure 3. The coordinate associated with the detector 
orthogonal pads are sufficient to locate the interaction point within a detector. Fig. 3 shows the coordinate system associated with the PSVFG detector. The pads response function gives the amplitudes of the pad signal versus the $X-Y$ coordinates of the electron cloud:

$$
A=R(x, y)
$$

Accordingly, the inverse $R^{-1}$ function allows us to evaluate $\mathrm{X}$ and $\mathrm{Y}$ coordinates. There are several ways to construct the inverse response function. We assume that the amplitudes from the opposite pads define the position of the line along X (or Y) directions, while the amplitudes from the two orthogonal pads determine the point location on the line. For example, if the position of the line along the $X$-axis is defined by $A_{x}^{1}$ and $A_{x}^{2}$ amplitudes, then we can have two estimates for $X$ where each depends on $A_{x}^{1}$ and $A_{x}^{2}$ :

$X_{1}=R^{-1}\left(A_{x}^{1}, A_{y}^{1}, A_{y}^{2}\right)$

$X_{2}=L-R^{-1}\left(A_{x}^{2}, A_{y}^{1}, A_{y}^{2}\right)$

where $L$ is a distance between two opposite pads. Combining both estimates we can write:

$X=\frac{X_{1} W_{1}+X_{2} W_{2}}{W_{1}+W_{2}}$

where $W_{l}$ and $W_{2}$ are the weights defined as:

$W=\frac{1}{\sigma_{x}^{2}}$

where $\sigma_{x}^{2}$ is the standard deviation of the estimates:

$\sigma_{x}^{2}=\left(\frac{\partial R}{\partial A}\right)^{2} \sigma_{A}^{2}$

The pad response functions have to be evaluated during the calibrations. In the first approximation, we can assume that they are identical for all detectors and all pads. We note that the above equations can be applied in the cases of using only 2- and 3-pad signals. In the case of 2 signals we have to use the amplitudes from two orthogonal pads and the exact response functions to evaluate single estimates for each direction ( $\mathrm{X}$ and $\mathrm{Y}$ ). In the case of 3 signals we have to use amplitudes from two orthogonal pads to evaluate a single estimate for one direction, and two amplitudes from the opposite pads to evaluate two independent estimates for another direction. The latter should be combined together using the weights. We note that for coordinate evaluations we always use relative to the anode pad amplitudes (uncorrected) which allows to use the same algorithm for all photon energies regardless the charge loss due to trapping.

Using a linear approximation for the response functions and taking the signal amplitudes as the weights, we can get a first-order estimate for $X$ and $Y$ coordinates:

$X=\frac{A_{x}^{\mathrm{1}}}{A_{\chi}^{1}+A_{\chi}^{2}}$

and

$Y=\frac{A_{y}^{1}}{A_{y}^{1}+A_{y}^{2}}$

The first order (linear) estimates can be used for correcting the response non-uniformity, but they cause notable distortions near the detector edges when used for evaluating the real-space coordinates, especially in the case of 2- and 3-pad signals. As we demonstrated [11] the second order response functions provide good position estimates with accuracy of less than $0.5 \mathrm{~mm}$ in $X$ $Y$ directions. For $Z$ coordinates we use a cathode to anode ratio, which gives less than $0.5 \mathrm{~mm}$. In 
all cases, the uncertainties in XYZ coordinates are primarily determined by the local variations of the electric field, which is a common problem of CZT detectors.

The second-order response functions can be iteratively evaluated using measured pads signals from an un-collimated ${ }^{137} \mathrm{Cs}$ source during the calibration. For each set of measured pad amplitudes (interaction event) we can use Eqs. $(7,8)$ to calculate the first-order approximations for $X$ and $Y$.

Another advantage of using PSVFG detectors is that we can correct their response nonuniformities caused by crystal defects and local variations of the electric field inside the detectors. Despite significant progress in the technology and capabilities of CZT detectors, crystal defects (which exist even in the best quality CZT crystals) still remains the major factor that limits availability and increases costs of actual CZT instruments. Therefore, the main challenge facing detector developers today is not how to overcome these problems.

The proposed high-granularity positionsensitive detectors allow for more accurate and efficient charge-signal corrections to overcome non-uniformities in the devices' responses.

Using the position information, we can virtually divide a detector volume into small voxels and correct (equalize) responses measured from each of them by using a 3-dimensional correction matrix generated during calibration. Fig. 4 illustrates the performance of a $20-\mathrm{mm}$ thick PSVFG detector before (left) and after (right) $\mathrm{X}-\mathrm{Y}$ corrections measured with uncollimated ${ }^{137} \mathrm{Cs},{ }^{60} \mathrm{Co}$, and ${ }^{133} \mathrm{Ba}$
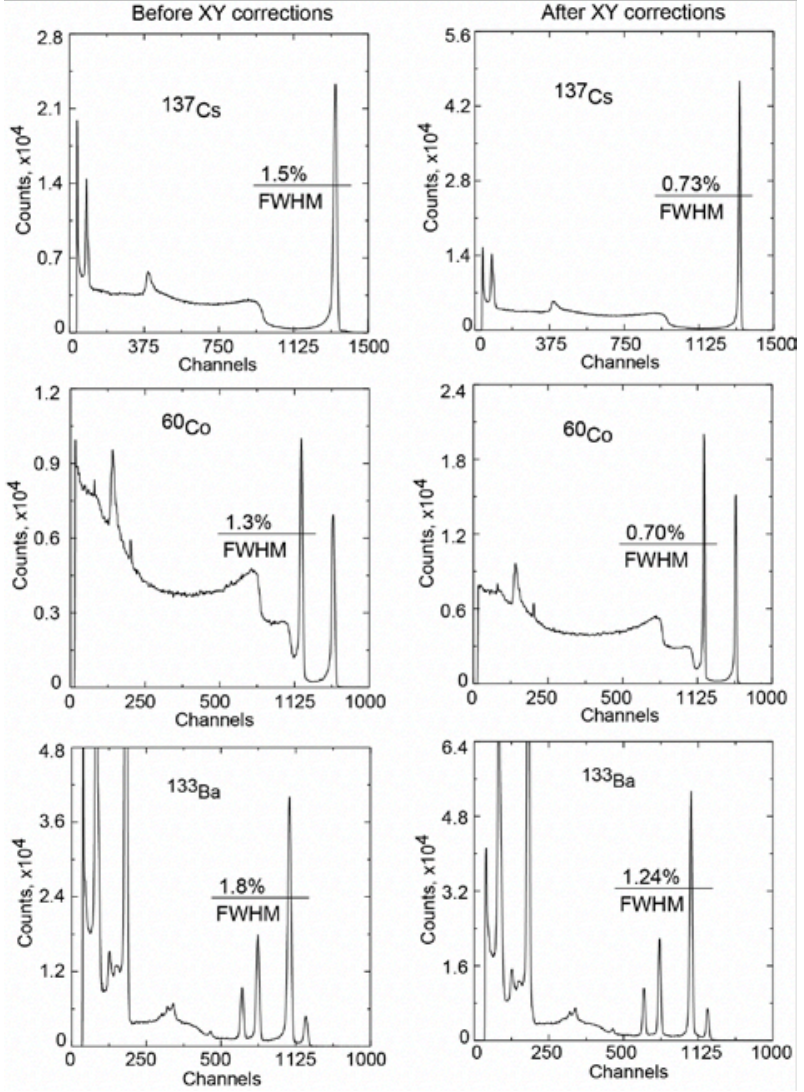

Figure 4. Performance improvements of a $20-\mathrm{mm}$ thick position-sensitive virtual Frisch-grid detector before and after corrections for three $\boldsymbol{\gamma}$-ray emitting sources [11] sources. However we applied the drift-time (or interaction depth) corrections to the spectra shown on the left [12].

The main factor limiting the energy resolution of the PSVFG detectors is electronic noise related to the high leakage current, 5-25 nA, and large anode capacitance, $\sim 3 \mathrm{pF}$. As we demonstrated, the detector's energy resolution stays below 1\% FWHM at $662 \mathrm{keV}$ for temperatures up to $\sim 30{ }^{\circ} \mathrm{C}$. Another potential problem in long-drift distance bars is the charge loss near the detector's side surfaces (edge effect).

These results demonstrate the feasibility of position-sensitive PSVFG CZT detectors and arrays of such detectors for high-resolution $\gamma$-ray spectroscopic measurements. After applying the 3D corrections, we achieved an energy resolution below 1\% FWHM at $662 \mathrm{keV}$ with a majority of the CZT crystals that we either grew at BNL or obtained from commercial suppliers. The ability 
to use the majority of CZT crystals should bring down the cost significantly, making this design useful in the manufacturing of commercial detectors.

\section{Concept of the Calorimeter}

The drift-grid CZT detector will enable a medium-energy $\gamma$-ray instrument to:

- provide incident photon energy measurement with high resolution, including the capability to measure cosmic nuclear lines;

- serve as a focal plane detector for Compton-scattered events to determine the direction and polarization of incident photons;

- enable a modular calorimeter of large area, which is critical for space instruments designed to measure low-intensity photon fluxes.

The base element of the calorimeter is a module made of 16 CZT bars, arranged in a $4 \times 4$ bar array. Bars currently being tested are $6 \times 6 \times 20 \mathrm{~mm}^{3}$, which makes the module dimension $2.5 \mathrm{x}$ $2.5 \times 2 \mathrm{~cm}^{3}$. The bars are encapsulated, tightly packaged and mounted on a printed circuit board (PCB). The module is read out by a single low-noise Application-Specific Integrated Circuit (ASIC) also positioned there (Fig. 5, left). The modules (as many as a particular instrument design requires) are plugged into the "mother" $\mathrm{PCB}$ tightly to each other, making the array of the required area with practically no "dead" space (Fig. 5, right). The "mother" PCB distributes the signals to the appropriate electronics placed on its sides (FPGA etc.).
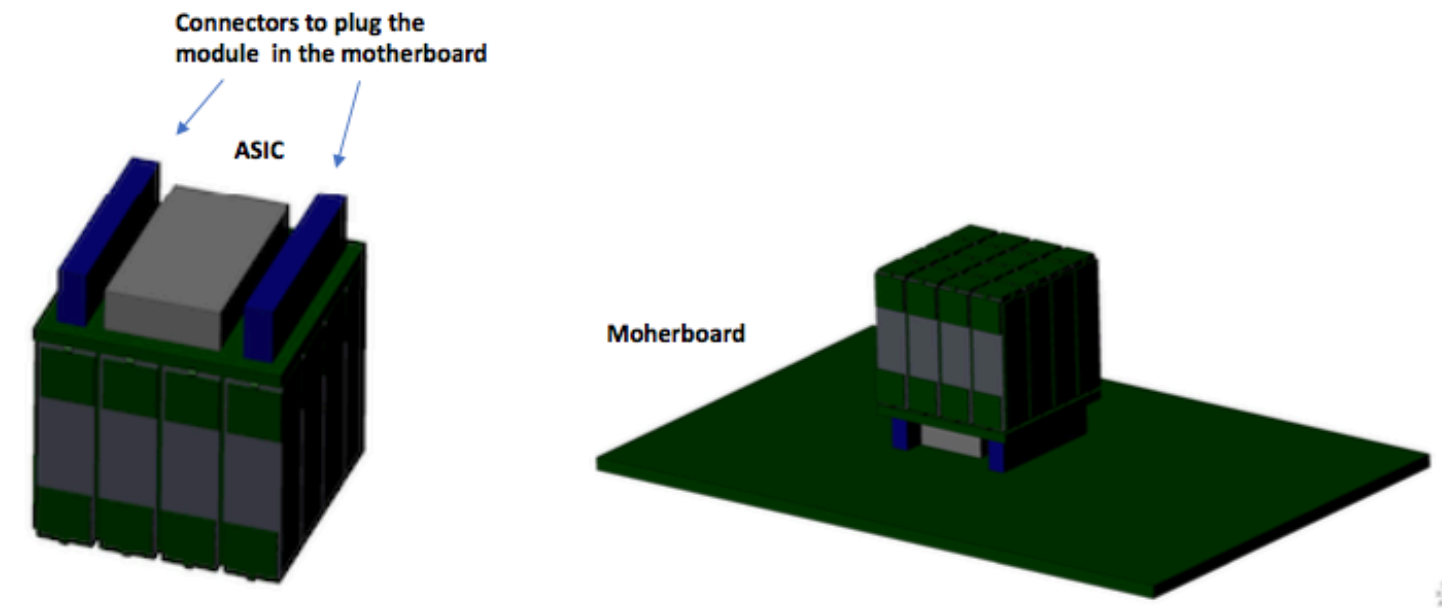

Figure 5. Individual 4x4 bar module (left, shown up-side-down) and plugged in the motherboard (right)

The best dimensions of the individual bars, and consequently the module size, are currently under investigation. Increase of the bar cross-section will reduce the number of needed bars to cover the same area, and also reduces the number of electronic channels. The cost of a larger size bar within these dimensions is roughly proportional to the detector volume, so the increased cross-section 
bar is probably the right approach. The increase of the bar length would be very good for the calorimeter performance because it would increase the detection efficiency (since the calorimeter thickness increases) and also the event containment. However longer bars experience larger leakage current and suffer from increased electric field distortion. Probably the optimal bar length is in the range of $30-35 \mathrm{~mm}$.

Ultimately we are targeting the following performance of the calorimeter:

a) extend detectable energy range down to $\sim 100 \mathrm{keV}$, limited by the readout electronic noise;

b) energy resolution better than $1 \%$ for the photon energy range $0.2-1 \mathrm{MeV}$ and better than $3 \%$ for 1-5 MeV. Operation in "pair-production" range (above $\sim 1.5 \mathrm{MeV}$ ) adds a problem of "escaping" annihilation $511 \mathrm{keV}$ photons, so requires more work on the design and data analysis

c) spatial resolution (point of the first interaction) $<1 \mathrm{~mm}$ for $\mathrm{E}<1 \mathrm{MeV}$ and 2-3mm for higher energy. Photons with energy $>\sim 1 \mathrm{MeV}$ have several interactions before being fully absorbed, so more work is needed to identify the point of the first interaction. Possible solutions are the use of waveform sampling ASIC and developing smart analysis software. The high spatial resolution of such a calorimeter can make viable its use in the instruments utilizing a coded aperture mask and focused on accurate study of densely populated sky areas, e.g. the Galactic Center or Cygnus region.

\section{Summary}

We are considering the use of such a calorimeter for the AMEGO Compton-Pair space telescope which is a NASA Probe-scale mission concept [1]. For that instrument the calorimeter array is $16 \times 16$ modules, making the area $\sim 40 \times 40 \mathrm{~cm}^{2}$. AMEGO will comprise four such arrays, bringing the total area to $\sim 80 \times 80 \mathrm{~cm}^{2}$. Extensive investigations are under way, which include numerous simulations, prototyping and testing. We are planning to run a beam test with photons of the energy $1-10 \mathrm{MeV}$ to test our concept at higher energies than can be achieved with radioactive sources, as well as environmental tests to validate the design for space applications.

\section{References}

[1] A. Moiseev et al., All-Sky Medium Energy Gamma-ray Observatory (AMEGO), $35^{\text {th }}$ ICRC, Busan, 2017

[2] A. E. Bolotnikov, et al., Use of high-granularity position sensing to correct response non-uniformities of CdZnTe detectors, Appl. Phys. Lett. 104, p. 263503, 2014.

[3] A. E. Bolotnikov et al., CdZnTe Position-Sensitive Drift Detectors with Thicknesses Up to $5 \mathrm{~cm}$, Appl. Phys. Lett. 108, p. 093504 (2016).

[4] Kaye, W. R., Energy and Position Reconstruction in Pixelated Cadmium Zinc Telluride Detectors. Ph. D. thesis, University of Michigan, 2012

[5] A. E. Bolotnikov et al., High-Efficiency CdZnTe Gamma-Ray Detectors IEEE Transactions on Nuclear Science 62, 3193, 2015. 
[6] V. V. Dmitrenko et al., Spectrometric applications of an ionization-type drift chamber, Pribory I Tekhnika Eksperimenta, vol. 1, pp. 51-53, 1982. Translation in Instruments and Experimental Techniques (USA), vol. 25, no. 1, pp. 50-53 (1982).

[7] K. Parnhamet al., Performance Improvement of CdZnTe Detectors Using Modified Two-Terminal Electrode Geometry, in Hard X-ray, Gamma-Ray and Neutron Detector Physics, Proceedings of SPIE Vol. 3786, pp. 49-54, 1999.

[8] C. Szeleset al., Fabrication of High Performance CdZnTe Quasi-Hemispherical Gamma-ray CAPtureTM Plus Detectors, Hard X-Ray and Gamma-Ray Detector Physics VIII, edited by Larry A. Franks, Arnold Burger, and Ralph B. James, Proceedings of SPIE Vol. 6319 (SPIE, Bellingham, WA, 2006).

[9] D. S. McGregor and R. A. Rojeski, High-resolution ionization detector and array of such detectors, US Pat. 6175120, 2001.

[10] G. Montemont et al., A Capacitive Frisch Grid Structure for CdZnTe Detectors, IEEE Trans. Nucl. Sci. 48, pp. 278-281, 2001.

[11] A. E. Bolotnikov et al., An array of virtual Frisch-grid CdZnTe detectors and a front-end applicationspecific integrated circuit for large-area position-sensitive gamma-ray cameras, Review of Scientific Instruments, vol. 86, no. 7, 2015. 